\title{
Colloque «l'économie verte au service de la croissance »
}

\author{
Étienne Pilorgé ${ }^{1, a}$ et Claude Roy ${ }^{2,3}$ \\ ${ }^{1}$ Cetiom (Centre technique interprofessionnel des oléagineux et du chanvre), Avenue Lucien Brétignières, 78850 Thiverval-Grignon, France \\ 2 CGAAER, Conseil général de l'alimentation, de l'agriculture et des espaces ruraux, 251, rue de Vaugirard, 75732 Paris Cedex 15, France \\ 3 Club des Bio-économistes, 4 allée du Progrès, 92170 Vanves, France
}

Reçu le 16 juillet 2013 - Accepté le 25 août 2013

\begin{abstract}
Résumé - Le 12 décembre 2012 avait lieu à Paris, au Centre de conférences du ministère de l'Économie et des Finances, un colloque sur «l'économie verte au service de la croissance » organisé par le CGAAER (Conseil général de l'alimentation, de l'agriculture et des espaces ruraux), avec l'appui des ministères chargés de l'industrie et de l'agriculture ainsi que de plusieurs partenaires, dont le «Club des Bio-économistes ». Organisé en tables-rondes, il se donnait alors pour objectif de mettre en valeur la contribution des bio-filières issues de l'agriculture, de la forêt et des bio-déchets, aux grands défis du futur (le fameux «monde fini »), en faisant le point sur les enjeux et les acquis en matière d'économie, d'innovation et de compétitivité.
\end{abstract}

Mots clés : Économie verte / bio-économie / carbone vert / bio-énergie

Abstract - "Green economy for global growth" conference. A conference about "green economy for global growth" organized by CGAAER (French General Council of food, agriculture and rural spaces), with the support of Ministries of Industry and of Food \& Agriculture, as well as of several partners, of which the "French Club of the Bio-economists" took place in Paris, in the center of conferences of the French Ministry of Finance, in December the 12th 2012. Round tables were organised in order to emphasize the contribution of the bio-sectors from agriculture, forest and bio-waste, in the great challenges of the future (the so-called "finished world"), by reviewing the challenges and achievements regarding economy, innovation and competitiveness.

Keywords: Green economy / bioeconomy / green carbon / bioenergy

Le 12 décembre 2012 avait lieu à Paris, au Centre de conférences du ministère de l'Économie et des Finances, un colloque sur «l'économie verte au service de la croissance» organisé par le CGAAER (Conseil général de l'alimentation, de l'agriculture et des espaces ruraux), avec l'appui des ministères chargés de l'Industrie et de l'Agriculture ainsi que de plusieurs partenaires, dont le «Club des Bio-économistes ».

L'économie verte, ou bio-économie, encore appelée l'économie du «carbone vert», comprend une dizaine de grandes filières de production/transformation : agro-industrie, transformation de la matière (bois, papier, panneaux, composites, oléochimie, bio-plastiques, xylo-chimie, fertilisants organiques, etc...), ainsi que les filières bio-énergétiques (biocarburants, chaleur, gaz, bio-électricité et cogénération,...).

La dynamique de l'économie verte ne relève plus aujourd'hui, comme au début des années 1980, de convictions principalement militantes mais bien de composantes économiques et stratégiques réelles, partagées par les opérateurs industriels, et dont on parle pourtant peu dans la presse. L'économie verte représente ainsi, en France, $5 \%$ des approvisionnements des industries de l'énergie, de la chimie et des matériaux,

\footnotetext{
a Correspondance : pilorge@cetiom.fr
}

contribution qu'il est prévu de doubler d'ici 2025 et qui pourrait représenter $20 \%$ ou plus des ressources en matières premières de l'économie française à l'horizon 2050. Cette ossature durable de notre développement compétitif constitue donc un vrai moteur de croissance et un choix sans regrets, ne seraitce qu'à cause du potentiel d'emplois très important qu'il soustend (70 000 emplois directs nouveaux, essentiellement territoriaux, ont été créés en 20 ans grâce à l'amorçage de ces nouvelles bio-activités).

Le colloque du 12 décembre, organisé en tables-rondes, se donnait alors pour objectif de mettre en valeur la contribution des bio-filières issues de l'agriculture, de la forêt et des biodéchets, aux grands défis du futur (le fameux «monde fini »), en faisant le point sur les enjeux et les acquis en matière d'économie, d'innovation et de compétitivité.

\section{Les enjeux : transition énergétique dans un cadre contraint et concurrences d'usages}

Claude Roy a d'abord posé les termes du débat : les grandes bio-filières préexistaient depuis l'antiquité ! Mais elles renouent avec le futur grâce à des changements majeurs, 
d'ordre technologique, mais surtout car il y a désormais 9 milliards d'habitants en vue sur la planète. Ceci nous fait toucher du doigt nos limites et prendre conscience que, face au défi climatique, nous ne disposons en réalité que de trois solutions pour agir et réagir : 1/ réduire nos consommations (sobriété), 2/ mettre le cap sur le renouvelable, 3/ favoriser la séquestration du carbone, notamment dans les sols, les forêts et les bio-produits. Or, la bio-économie est la seule qui puisse embrasser simultanément et positivement ces trois axes vertueux, avec en outre des coûts modestes et de multiples externalités positives !

Mais cela demande en particulier de produire beaucoup, en agriculture comme en forêt, tout en gérant et en mobilisant efficacement toutes nos bio-ressources, afin notamment de prévenir et de maîtriser les risques de concurrences d'usages entre les bio-filières. D'où des questions de gouvernance, d'équilibres et de synergies!

Patrice Geoffron (université Paris Dauphine) pointe alors le défi de la transition énergétique, qui est à relever en un temps contraint et avec une population croissante. Il remarque que les transitions énergétiques précédentes de notre histoire reposaient toutes sur un avantage lié à la maîtrise d'une ressource énergétique nouvelle et massive : le charbon, le pétrole... Or, nous ne sommes plus dans une telle logique! L'énergie reine reste bien sûr le charbon par ses volumes encore disponibles. Mais les perspectives sont finies. Il y a certes une persistance assurée à court et moyen termes des bouquets de ressources d'énergies fossiles, avec trois siècles de consommation actuelle de charbon, un siècle de gaz, et plusieurs décennies de pétrole (sans même évoquer les réserves de gaz et pétrole de schistes, une ou deux décennies), mais c'est bien peu... Et c'est pourtant « trop » par rapport aux contraintes climatiques que leur combustion ne ferait qu'aggraver, bien au delà du « scénario de l'inacceptable». Dans ce contexte de transition énergétique risqué et plein d'incertitudes, la biomasse présente des avantages notables, notamment pour l'Europe. D'abord du point de vue de la sécurité des approvisionnements, puisqu'elle permet, contrairement à toutes les autres filières renouvelables, d'alimenter aussi les marchés des hydrocarbures (bio-carburants) et de la chimie. Ensuite, du point de vue de la complémentarité des énergies : la biomasse ne présente pas l'inconvénient de l'intermittence, contrairement aux autres filières renouvelables (éolien, hydraulique, solaire...). Elle se stocke! Enfin, du fait du contenu élevé en valeur ajoutée et en emplois «territoriaux» de la «bio-économie». Mais ces nouvelles bio-filières ne seront pleinement compétitives que si l'on peut valoriser et monétariser un certain nombre de facteurs, au premier rang desquels la valeur en $\mathrm{CO}_{2}$ «évité », ce que l'actuel marché du carbone n'assure pas. Cela repose en particulier la question des taxes carbone..

Pour Pierre Radanne (président de l'Association 4D ${ }^{1}$ et ancien président de l'Ademe ${ }^{2}$ ), la transition énergétique dans

\footnotetext{
1 Association Dossiers et Débats pour le Développement Durable, créée en 1993, après le sommet de Rio, afin de constituer un réseau citoyen pour la promotion du développement durable et le suivi des engagements pris par la France, comme par les autres États membres de l'ONU.

2 Agence de l'Environnement et de la Maîtrise de l'énergie.
}

un tel calendrier contraint ne pourra être assurée uniquement avec des technologies et des améliorations d'organisation collective. Il faudra aussi jouer sur des attitudes personnelles nouvelles, ce qui suppose un investissement fort sur la personne, ce qui constitue une rupture. Le débat national sur l'énergie intervient au moment où l'on sort d'un cycle énergétique (le pétrole d'après-guerre, les chocs pétroliers des années 1970 et le nucléaire...) et où les équipements en place se rapprochent de leur fin de vie. Les décisions stratégiques doivent alors, pour la première fois de notre histoire, être prises dans un contexte d'augmentation durable des prix de l'énergie fossile, et avec le changement climatique en toile de fond. La question du changement climatique nécessite de diviser par 2 nos émissions planétaires de gaz à effet de serre d'ici 2050 (et par 4 dans les pays des développés, dont la France), car au-delà de $+2{ }^{\circ} \mathrm{C}$ de réchauffement moyen mondial, outre tous les autres dérèglements imaginables, un problème alimentaire général risque notamment de survenir, avec des pertes considérables de rendements agricoles dans certaines régions du monde. L'implication du citoyen dans le débat est donc d'autant plus importante qu'il est acteur et consommateur de l'énergie et des autres matières premières «fondamentales », l'eau et l'alimentation.

Le débat national sur l'énergie comprend, en France et en Europe, deux composantes : comment l'énergie peut-elle contribuer à l'équilibre du pays ? Comment mener une transition énergétique alors que les caisses sont vides? Avec deux choix possibles : repousser les investissements, mais en sachant que cela gonflera entre temps les importations et aggravera notre dépendance géo-stratégique, ou bien programmer des investissements qui soient gagés par la baisse des dépenses et donc par la baisse de la facture énergétique.

Que se passe-t-il dans les autres pays du monde? Tous ont de fait le même calendrier (pour les pays industrialisés), sachant que les pays émergents sont en phase de construction.

Au niveau mondial, un nouvel accord sur le changement climatique a été signé à Doha, a minima et peu satisfaisant. Il opérera jusqu'en 2020, avec une période transitoire, mais en ne portant que sur un engagement à limiter le réchauffement planétaire moyen à $+4{ }^{\circ} \mathrm{C}$ d'ici la fin du siècle, alors même que nous savons que de graves problèmes surgiront au-delà $\mathrm{de}+2{ }^{\circ} \mathrm{C}$. Il existe une rupture très forte entre l'Amérique du Nord et l'Europe, et plus généralement entre les pays « vides » (grandes surfaces, ressources importantes, populations peu denses) et les pays «pleins » (situation inverse, comme en Europe) : les pays «pleins » se doivent d'être «polis » avec les autres, les «vides », qui n'ont pas de problèmes de ressources. Restent les émergents : l'Inde et la Chine, qui sont aussi des pays «pleins », quoiqu'immenses. C'est de fait l'Europe, sans ressources, qui va être obligée d'animer la construction du futur. Une conférence est prévue à Paris en 2015 pour chercher un accord «climatique » à 193 pays. Les pays en développement ont désormais compris qu'ils ne pourraient pas accéder à la trajectoire de développement qu'ont connu les pays développés... Peut-être, alors, un accord sera-t-il possible, malgré la crise, et malgré les écarts de développement?

Pierre Radanne note incidemment que la notion de souveraineté nationale est morte à Copenhague : les souverainetés nationales ne garantissent plus en effet l'intérêt général, 
notamment climatique. Elles doivent donc se plier devant l'ONU. L'enjeu principal porte alors sur une énorme transformation de la gouvernance du monde, avec d'un côté les pays «vides», et de l'autre les pays «pleins ». Sur ces aspects, l'Europe est particulièrement innovante en ayant notamment construit une «souveraineté climatique transnationale». Mais la question va être, au niveau mondial, de fixer des engagements avec des objectifs temporels définis, dans l'équité, et avec des règles du jeu pour le respect des accords. L'accord à conclure pour 2020 devra donc être fondamentalement nouveau, et il déterminera les conditions de la paix dans ce siècle.

Patrice Geoffron, en complétant ce qui précède, remarque que la position de l'UE est troublante : plutôt efficace et sobre en émissions de $\mathrm{CO}_{2}$, l'Europe avait annoncé pouvoir aller sous certaines conditions jusqu'à $30 \%$ de réduction d'émissions de gaz à effet de serre, au lieu de $20 \%$. Mais passer de $20 \%$ à $30 \%$ au niveau de l'UE ne représente en fait que le gain de deux semaines d'émissions chinoises... Si le modèle à suivre de la croissance verte est bien en Europe, comme le dit Pierre Radanne, et si l'UE peut permettre à tous de gagner du temps, elle ne peut quand même pas sauver le monde seule... !

Marc Roquette (groupe Roquette Frères) s'est exprimé sur l'enjeu particulier de la chimie du végétal, que l'on oublie souvent dans les débats faute d'être un enjeu «énergétique », alors que $15 \%$ de la consommation mondiale de pétrole va à la chimie ! La migration de la chimie du pétrole vers la chimie du végétal mettrait en jeu annuellement deux milliards de tonnes de matières premières, ce qui constituerait une pression très forte sur les ressources en biomasse disponibles. Ceci amène notamment à réfléchir aux synergies et aux arbitrages dans l'usage des bio-ressources avec les bioénergies et les biocarburants, comme avec l'alimentation, (d'autant que d'un point de vue énergétique, une surface terrestre productive est mieux valorisée par l'éolien - malgré l'intermittence de production, handicapante - que par les biocarburants). La migration du pétrole vers le «pétrole vert» en chimie a commencé avec l'éthanol, désormais presque entièrement produit sur base canne à sucre/betteraves.

Mais le développement de la chimie verte rejoint la question énergétique sous un autre aspect, car la fabrication de produits sur base végétale requiert beaucoup d'énergie. Pour fabriquer en base végétale sans énergie fossile et sans émissions de $\mathrm{CO}_{2}$, des investissements sont nécessaires : géothermie, méthanisation des eaux résiduaires... Coupler la chimie verte et le nucléaire peut aussi être une solution (comme pour le BtL caburant), mais le solaire représente aussi un flux technologique considérable $(+10 \% / \mathrm{an})$. Pour progresser, la taxation des énergies apparaît nécessaire, et l'UE aurait une possibilité pour construire un business model différent de celui des USA. Marc Roquette suggère ainsi de mettre en place un système de «prélèvements et de restitutions énergétiques », jusqu'à 100 \$/baril, à l'image des prélèvements et des restitutions agricoles qui ont permis le développement de l'agriculture européenne, en rappelant que le groupe Roquette a jadis travaillé avec du maïs trois fois plus cher que le marché mondial... mais dans le contexte de l'ancienne PAC, avec des rééquilibrages aux frontières. Ce business model différent permettrait de faire des économies bien plus importantes qu' avec le gaz de schiste européen.
En matière de nutrition-santé issus de la biomasse, l'évolution se fera au bénéfice des protéines végétales que les technologies permettront de rendre plus appétentes, avec en outre un enjeu pour la conversion des sucres en protéines.

Jacques Valeix (ONF, Office national des forêts) a proposé de son côté une traduction de ces enjeux pour le secteur de la forêt, non sans rappeler les incertitudes liées au changement climatique. Il remarque que, d'un point de vue économique et social, la transformation des bois français reste toujours délocalisable (en valeur ajoutée) et que les possibilités d'évolution de la filière bois française méritent une grande attention dans le contexte de transition énergétique et écologique. Il souligne l'importance de mieux sécuriser l'approvisionnement en bois des industries de transformation, avec des logiques 1/ d'aménagement, 2/ de hiérarchisation des usages (bois d'œuvre, bois d'industrie, bois énergie), 3/ d'investissements en vue d'améliorer la valeur ajoutée générée sur le territoire. Toutes ces actions reposent sur la capacité à conforter notre modèle économique de la gestion durable (y.c. productive) des forêts en en rétribuant en outre les aménités positives (stockage de carbone, qualité de l'eau...) dans la mesure où les produits de la vente du bois ne peuvent pas couvrir seuls l'ensemble des coûts des fonctions d'intérêt collectif de la forêt.

Fabien Bova, directeur général de FranceAgriMer, a complété l'analyse pour le secteur agricole en mettant en avant trois aspects :

- 1) la hiérarchisation des usages des bio-ressources : l'alimentation humaine et animale doit rester prioritaire sans ambiguiité, suivie des bio-matériaux et de la chimie verte, et enfin des usages énergétiques (dont les biocarburants), le tout en recherchant toujours les meilleures synergies possibles entre filières (ex. biocarburants de génération 1 et tourteaux pour l'alimentation animale).

- 2) l'observation des concurrences d'accès aux bioressources : dans la mesure où l'on observe des concurrences fortes entre bio-filières, il y a un véritable enjeu dans l'observation des évolutions en termes d'utilisation et de règles d'accès aux marchés pour les différentes bioressources et leurs différents usages.

- 3) la concurrence alimentaire/non alimentaire : le constat est que pour satisfaire les objectifs «biocarburants » de la directive ENR 2020, l'UE mobiliserait $7 \%$ de ses surfaces agricoles utiles, surfaces à diminuer peut être de moitié pour prendre en compte les co-produits venant en feed-back et qui sont dévolus à l'alimentation animale (génération 1). Or, l'impact de cette directive par rapport à l'enjeu climatique global et à l'indépendance énergétique européenne restera faible. En revanche, les conséquences en termes de poussée des prix des matières premières agricoles peuvent ne pas être négligeables. On observe déjà des tensions!

Depuis 10 ans, on constate une augmentation structurelle de la volatilité des prix sur les marchés agricoles et une course à la compétitivité. Cette concurrence semble encore plus forte, et plus internationale, dans les usages énergétiques, avec des groupes mondiaux, peu de contraintes sanitaires, et des diversifications croissantes dans les marchés. L'ingéniosité des 
transformateurs et des chimistes fait en outre qu'il est très difficile de maîtriser les barrières tarifaires avec ce type de produits.

Quant à la France, elle est assise sur une très bonne production agricole mais elle présente des performances industrielles un peu moins compétitives. La réflexion collective en filières est importante (Quelles matières premières? Quel est le meilleur rendement technologique? Quel est le meilleur «marketing mix»? Quelles évolutions réglementaires promouvoir?).

\section{Les efforts passés et les acquis technologiques, organisationnels et humains}

Pour Jean-Claude Sabin, fondateur de PROLEA, et précurseur de la bio-économie en France, la montée en puissance, y compris énergétique, de la filière des oléagineux et protéagineux s'est au départ construite à partir d'objectifs de reconquête des marchés des protéines, notamment à la faveur du Plan Protéines en 1973, le colza apparaissant alors comme un « vrai soja européen » selon Jacques Poly. Jean-Philippe Puig, directeur général de Sofiprotéol, souligne en effet l'importance des protéines comme enjeu au niveau de la planète, mais aussi pour l'Europe (70 \% d'importation) et pour la France, et le rôle moteur de la vente des tourteaux riches en protéines pour le développement du biodiésel. Le biodiésel a lui-même généré un autre co-produit industriel, la glycérine, qui est l'un des produits « socle » qui permettent de développer la chimie du végétal aujourd'hui. Sofiprotéol est devenu leader mondial de la production de glycérine avec ses entreprises Oléon et Novance. Dans une optique d'économie circulaire, les investissements se sont poursuivis dans les filières animales. Aujourd'hui, le groupe compte 8000 personnes contre 400 il y a 10 ans. Ce développement en filière repose sur l'innovation et sur l'accélération du processus de Recherche et Développement pour gagner du temps. Au-delà du groupe industriel, Sofiprotéol est un groupe financier, qui investit dans les biocarburants de première, de $2^{\mathrm{e}}$ et de $3^{\mathrm{e}}$ générations. La deuxième génération est prévue pour 2020-2025 au stade industriel, et la R\&D est organisée autour du pilote BioTfuel (conversion de la biomasse ligno-cellulosique en carburants de synthèse), projet encore unique au monde à ce stade : la France est ainsi, aujourd'hui, probablement leader sur la deuxième génération «thermochimique » des biocarburants et de la chimie du végétal.

Jean-Philippe Puig, estime qu'en technologie, et même en prix de revient, Sofiproteol n'a rien à envier en effet à quiconque, mais le soutien que peut apporter l'Etat à une telle filière d'avenir, et surtout la fiabilité et la visibilité de ces engagements publics auprès des filières, font in fine la différence. On voit en outre arriver sur les marchés des produits estérifiés « dopés financièrement», plus ou moins entrés légalement aux frontières de l'UE, moins chers que le prix de l'huile (ex. origine Argentine...)! La distorsion de concurrence nuit au développement des filières, et il faut à l'évidence protéger ce développement car il abrite beaucoup de valeur ajoutée, d'indépendance énergétique et d'emplois en France !

Aujourd'hui, le biodiesel est incorporé au diésel à la pompe, en France, à hauteur de $7 \%$, avec une moyenne de
4,75\% en UE. Des débats ont lieu pour revoir à la baisse l'objectif européen qui avait été fixé à $10 \%$ en 2020 par la directive UE de 2009. En France, le ministère chargé de l'Agriculture a décidé de «faire une pause à $7 \%$ », mais l'outil industriel français a d'ores et déjà été dimensionné pour atteindre les $10 \%$. Il est donc important de poursuivre ce développement quelques années encore car c'est bien la première génération de biocarburants qui financera et permettra (si c'est possible) d'accéder aux $2^{\mathrm{e}}$ et $3^{\mathrm{e}}$ générations ${ }^{3}$.

Parmi les pionniers de la bio-économie française, il y a vingt ans, et avec Jean Claude Sabin, Régis de Baynast, fondateur de $\mathrm{ARD}^{4}$, a rappelé l'importance du projet de la nouvelle bio-économie : les biocarburants, considérés au départ comme un co-produit, ont connu d'abord l'opposition des pétroliers. À l'opposé, la «bio-cosmétique » est apparue très vite comme un projet consensuel : Les détergents et les tensioactifs bio-sourcés ont ainsi constitué rapidement un champ porteur, dans la cosmétique et la chimie. L'idée d'origine était simple : les végétaux ont développé des fonctions spécifiques pour germer et croître... on voulait extraire ces fonctions ! Et il importait également de faire travailler toutes ces filières ensemble pour créer des synergies.

L'importance du facteur humain et du pari sur l'avenir été rappelée par Jean-Marie Gabillaud, président de Coop de France Développement Durable : l'économie verte se fera avec les hommes et les femmes présents sur les territoires, qu'il faut veiller à ne pas décourager (attaques et remise en cause des pratiques agricoles, changements de cap dans les stratégies publiques, etc.) dans un contexte démographique agricole à la baisse et face à une urbanisation dominante. L'exemple du développement de l'activité bio-matériaux à la Cavac (Coopérative agricole Vendéenne d'approvisionnement, de ventes de céréales et autres produits agricoles) trouve son origine chez les agriculteurs eux mêmes, dans une réflexion collective à partir d'une problématique de rotation des cultures, et de parasitisme (orobanche sur le colza). Cela a amené à développer le lin oléagineux, et au final à cultiver 2500 ha de lin et de chanvre pour alimenter une usine de bio-matériaux d'isolation. L'expérience a permis d'identifier d'autres partenaires et des concurrents, et a ouvert d'autres perspectives, avec d'autres métiers (rénovation économe et durable du bâti). Les coopératives ont l'avantage de rassembler les agriculteurs. Le territoire français représente $5 \%$ de la SAU mondiale, parmi les meilleures terres, avec un vrai devoir de productivité, de production et d'exportation face à la démographie mondiale : la France doit donc aussi se lancer résolument dans cette partie de la production non-alimentaire.

Le modèle coopératif existe dans toutes les activités, et peut s'appliquer au domaine de l'énergie, de la production à la distribution. Devant le constat que les coopératives agricoles

\footnotetext{
3 En juillet 2013, Sofiprotéol, décide de réorganiser ses activités biodiesel en raison des difficultés rencontrées dans le secteur des biocarburants et prévoit la fermeture de deux unités de production de biodiesel, représentant une capacité de production de 380000 tonnes et d'une unité de trituration, pour adapter sa capacité aux objectifs de $7 \%$.

4 Agro-Industrie Recherches et Développement, société privée de
} recherche. 
ont en partie perdu dans le passé la bataille de l'intégration de la valeur ajoutée et de la consommation des produits alimentaires, il doit importer aux coopératives d'être en revanche, dès aujourd'hui, des maillons importants de cette nouvelle chaîne de valeur de l'économie verte, et de se mettre en capacité de conserver au maximum cette valeur ajoutée sur les territoires.

Selon Luc Charmasson, président de l'interprofession du bois-fibres «FBIE», France Bois Industrie Entreprises, la forêt française, troisième d'Europe, présente un fort potentiel de développement. La forêt participe à la biodiversité, par nature, et ce depuis des millénaires malgré (ou peut être à cause de...) sa mise en valeur constante par l'homme au cours des siècles (il n'y a plus de «forêt vierge » en Europe, et pourtant, la forêt y est particulièrement bien protégée, car elle est mise en valeur... ). Mais la forêt est surtout le «bras armé » de la prévention climatique, avec le stockage photosynthétique de carbone, qui se voit prolongé à travers les produits en bois (1 T $\mathrm{CO}_{2} / \mathrm{m}^{3}$ ), lesquels se substituent sobrement et fonctionnellement à des produits énergivores, tels le béton ou l'acier.

La filière bois française compte actuellement 190000 emplois en amont et 250000 en aval, avec 80 milliards $€$ de chiffre d'affaires, dont 20 pour l'amont. La question, c'est la dynamique de la ressource forestière, de sa mobilisation et de son renouvellement, notamment pour contribuer à prévenir le risque climatique. On connaît l'intensité insuffisante de la gestion actuelle de la forêt française, qui reste peu efficace en ne collectant que $60 \%$ de la ressource annuelle. Mais la France a également baissé la garde sur le reboisement depuis 20 ans, avec le risque «d'asphyxier à terme » la pompe à carbone forestière et de ne pas pouvoir renouveler ses ressources, notamment résineuses! Nous ne mettons en place que 50 millions de plants forestiers par an pour 300 millions en Allemagne. Or, il y a besoin de 20 millions de $\mathrm{m}^{3}$ /an supplémentaires en 2020 pour le seul bois énergie, sans compter les demandes croissantes en bois d'œuvre et d'industrie (notamment résineux), en particulier pour la construction et la rénovation « durables et énergétiquement sobres ». Le développement du bois énergie passera d'ailleurs par celui du bois d'œuvre et du bois industrie. Il faut hiérarchiser les usages, avec des valeurs ajoutées créées qui vont de 1 pour les filières de l'énergie, à 5 pour celles du bois d'oeuvre. Enfin, la forêt française devrait évoluer vers un accroissement des nouveaux peuplements résineux, comme en Allemagne, (peuplements plus productifs et plus efficaces en stockage de $\mathrm{CO}_{2}$ ), dont les marchés sont structurellement demandeurs, alors que l'offre française de bois reste très majoritairement feuillue (contrairement à l'Allemagne), avec des débouchés plus difficiles à entrevoir pour l'avenir.

Pascal Dupuis, chef du service Climat et efficacité énergétique du ministère de l'Écologie, du Développement durable et de l'Énergie, a dressé le tableau des acquis en matière de politiques et de réglementation. De son point de vue, si les avancées de Doha («round climat») ont été qualifiées par la presse de modestes, avec des engagements a minima, elles ont été utiles dans la mesure où elles dégagent la négociation à venir d'un certain nombre de contentieux « de base » qui ont été effacés.

Dans le domaine de l'énergie, le paquet énergie-climat (2008) constitue une base claire au niveau européen, mais la pression doit aller d'abord à la maîtrise de la consommation énergétique. Aujourd'hui 160 millions de TEP/an d'énergie sont consommées en France. En 2007, les énergies renouvelables en représentaient 17 millions de TEP (soit plus de $10 \%$ ), dont 14 en biomasse (et dont 10 en bois énergie). L'engagement pris dans le cadre du paquet énergie climat est d'augmenter encore la production de ces énergies renouvelables d'ici 2020 de +20 millions de TEP/an supplémentaires, dont plus de la moitié à partir de la biomasse ! Pour atteindre ces objectifs en 2020, nous devrons aussi réduire à 130 millions de TEP nos consommations (soit $-20 \%$ ), avec alors une perspective possible de $30 \%$ de renouvelable. Des outils sont mis en place : outils fiscaux pour les biocarburants, tarifs et appels d'offres pour la partie électrique, fonds chaleur géré par l'ADEME pour les chaufferies biomasse industrielles et collectives, et crédits d'impôt aux particuliers.

Dans le portefeuille renouvelable, la mise de fond principale porte sur la chaleur et sur la biomasse (bien que l'on parle plus volontiers de solaire et d'éolien électriques, qui resteront pourtant limités) : l'électricité ne représente plus que $23 \%$ de la consommation énergétique nationale. Et la chaleur est aussi la voie la plus efficace vis-à-vis du renouvelable, et la moins coûteuse en soutiens publics (à l'échelle du renouvelable), à hauteur de $3 € / M W h$ soit de 10 à 100 fois moins que pour les autres sources renouvelables, électriques notamment.

Les points de vigilance au niveau des politiques doivent porter sur :

- la nature du fond chaleur, qui est un poste de dépense budgétaire, et du crédit d'impôt, qui est une niche fiscale... objets d'examens budgétaires annuels et donc toujours susceptibles de remise en cause. Or on sait bien que les «stop and go » en matière de politique énergétique, et notamment de bio-énergies, sont toujours extrêmement longs et coûteux à « rattraper ».

- La hiérarchie des usages parmi les bio-ressources : les soutiens aux politiques bio-énergétiques (et les différentiels de tarifs électriques aux frontières...) sont d'une efficacité redoutable, mais peuvent entraîner des dérives : il ne faut pas en arriver à «brûler le bois d'œuvre ou le bois d'industrie », d'autant que du point de vue de l'emploi, les énergies renouvelables sont largement moins utilisatrices de main d'œuvre, et créatrices de valeur ajoutée, que le bois d'œuvre ou d'industrie.

- L'organisation de la vie du matériau avec plusieurs étapes : bois matériau, puis ressource pour l'industrie, avec notamment recyclage, puis combustible de récupération in fine.

- Le suivi des impacts : brûler de la biomasse s'avère très favorable en termes de $\mathrm{CO}_{2}$ et d'énergie, mais cela peut générer aussi d'autres impacts sur la qualité de l'air : $38 \%$ des particules atmosphériques sont aujourd'hui issues de la biomasse, notamment dans les utilisations domestiques plus ou moins bien maîtrisées. On ne brûle pas en effet, malheureusement, que du bois «propre et sec» issu de la forêt dans les chaudières et les cheminées, mais aussi, bien souvent, des résidus de bois humides, ou des déchets de bois par exemple, qui peuvent être adjuvantés... On essaie alors de clarifier et de normaliser les conditions dans lesquelles tout ceci est brûlé, et de mettre en place des filières. 
Un débat national sur la transition énergétique est nécessaire et va être ouvert. Les politiques publiques doivent viser la sobriété en même temps que l'efficacité, en définissant le mix-énergétique optimal et en traitant les questions du financement et de la fiscalité écologique.

\section{L'innovation}

Daniel Thomas, vice-président du pôle de compétitivité Industries et Agro-Ressources, a présenté, dans le cadre de l'enjeu de l'innovation, la problématique de la bio-raffinerie. Pour valoriser l'ensemble des bio-ressources disponibles, il faut utiliser des procédés beaucoup plus respectueux des composants complets de la plante. Cela permet d'avoir un modèle économique plus intéressant, basé sur une véritable gamme de valorisation optimisée des mêmes ressources : ingrédients alimentaires, cosmétique, bio-matériaux, chimie... et énergies (hydrocarbonées, calorifiques, gazeuses, électriques...)

L'IEED $^{5}$ «PIVERT » travaille ainsi pour une bio-raffinerie adaptable et évolutive sur les oléagineux. L'objectif est de concevoir une bio-raffinerie très flexible, à l'image de la programmation linéaire des industriels pétroliers dans leurs propres raffineries. Est-il facile de remplacer les produits chimiques pétroliers? Il est incontestable que les molécules pétrolières ont un niveau «énergétique » et un stade «d'évolution géologique» plus élevé, mais pour atteindre les produits de la chimie fine, il faut franchir d'énormes barrières d'énergie d'activation. Celles-ci sont plus faibles dans le domaine de la biomasse, et il est possible de renforcer encore cet avantage par des processus biotechnologiques.

Les évolutions actuelles de notre bio-économie consistent à aller vers plus de diversité dans les plantes entrantes, à mieux utiliser l'ACV (analyse du cycle de vie) comme indicateur et à développer l'ancrage au territoire : la bio-raffinerie doit se concevoir comme une activité intégrée à son écosystème.

Jean-Marc Louvet, président d'UPM France (UPM comprend trois groupes d'activité : énergie \& pâte, papier et matériaux évolués), a présenté à son tour les voies de développement et d'innovation de l'industrie du papier-carton, basées sur la diversification des valorisations de la biomasse lignocellulosique dans des matériaux composites, des bio-produits et des carburants/chimie de deuxième génération (notamment thermo-chimiques). Une unité industrielle a ainsi été lancée en 2012 en Finlande (centre de production et centre de recherche) pour utiliser les sous-produits de la fabrication de la pâte à papier. Un autre projet très important de niveau industriel est prévu en France, à Strasbourg. Les bio-carburants de deuxième génération permettront notamment de donner de la valeur supplémentaire à la filière «pâtes-papiers » dans la mesure où certains de ses sous-produits ligno-cellulosiques ne sont pas exploités aujourd'hui. La possibilité d'atteindre $80 \%$ de réduction des gaz à effet de serre émis par rapport à une base de référence fossile pourrait être envisagée. Il s'agit là d'innovations de rupture, stratégiques, et de portée mondiale, qui ne touchent pas que les procédés.

George-Henri Florentin, directeur général de l'institut FCBA (institut technologique Forêt Cellulose BoisConstruction Ameublement), a constaté de son côté le retard

\footnotetext{
5 Institut d'excellence des énergies décarbonées.
}

de la France où les produits de construction bois représentent moins de $10 \%$ du marché, contre $15 \%$ en Allemagne et 30 $\%$ aux USA. Selon lui, le problème va bien au-delà de la seule question de l'innovation dans la construction, dans la mesure où, face à l'ancrage du béton et de l'acier dans nos cultures et nos savoirs-faire, les habitudes d'utilisation du bois ont été perdues. L'affaiblissement des connaissances a entraîné alors la méfiance vis-à-vis de techniques pourtant séculaires (ex. colombages) mais oubliées. Lever ces freins passe par de la formation et de la qualification, notamment chez les bureaux d'étude, mais aussi par un travail d'information vers les « réglementeurs » et les normalisateurs, tant dans la construction neuve que dans la réhabilitation.

Le travail sur l'innovation et pour la levée des freins au développement du bois en construction repose quant à lui également sur les caractéristiques du matériau : le bois a d'extraordinaires propriétés mécaniques en flexion, ce qui constitue par exemple un avantage par rapport au béton. Au niveau des propriétés thermiques, pour viser les $50 \mathrm{Kwh} / \mathrm{m}^{2} /$ an de performance énergétique, le bois s'avère un très bon isolant, mais les spécialistes considèrent en revanche que ses caractéristiques ne sont pas idéales en été : c'est là un point à travailler. La durabilité du matériau du fait des champignons et des insectes est un autre point délicat car l'imprégnation chimique du matériau ne doit pas être défavorable à la santé et à l'environnement. Des travaux sur la conception sont aussi nécessaires (un escalier en extérieur ne se crée pas comme un escalier intérieur...), tout comme ceux permettant d'utiliser la chimie, les traitements thermiques (qui peuvent permettre de mieux valoriser les bois feuillus), les nouvelles colles sans solvants, etc.

Selon Yves Robin, chef de service de l'industrie du ministère du Redressement Productif, d'un point de vue politique, on aurait pu faire un système à part de la bio-économie, qui irrigue une part considérable de l'industrie : bois, papier, textile, agro-industrie... Cela aurait sans doute été une erreur en matière d'innovation. Il nous a semblé préférable de mobiliser l'existant : l'Ademe, qui a été sollicitée pour renforcer les programmes dans ce domaine, les pôles de compétitivité, les programmes de l'ANR (Agence nationale de la recherche), d'Oseo (établissement public dont la mission est de soutenir l'innovation et de la croissance des PME; Oseo est devenu BpiFrance le 12 juillet 2013) et quelques autres dispositifs plus ciblés, dont les «Investissements d'Avenir » avec plusieurs axes, et les instituts d'excellence sur les énergies décarbonées (IEED), ... etc. L'idée est bien de structurer l'ensemble de la démarche bio-économique face à la diversité de ses marchés et concurrences.

Daniel Thomas a répondu en soulignant les évolutions considérables de l'économie bio-sourcée depuis 8 ans : jusqu'en 2006, les programmes de recherche européens mobilisaient peu le monde industriel. Depuis, les industriels se sont fortement impliqués. Le pôle IAR (pôle de compétitivité Industries et Agro-Ressources) a ainsi monté et fait labelliser pour 1 milliard d'euros de projets avec deux tiers de financements privés. Les acteurs du domaine, au premier rang desquels les agro-industriels, ont fait des efforts considérables et les chimistes ont suivi. On est entré dans une période très significative de réalisations industrielles. Mais la France et l'Europe 
ont un handicap : certains pays tiers co-financent en effet la première réalisation industrielle avec des fonds publics. Ce n'est pas le cas en Europe ! Mais il y a en revanche des interventions régionales très fortes, qui permettent d'espérer fixer sur le territoire français des innovations stratégiques.

Sur les aspects de financement, Laurent Arthaud (CDC Entreprises) a exposé la place de la bio-économie dans les choix de financements de CDC Entreprises, filiale de la Caisse des Dépôts. CDC-E investit tout au long de la chaîne d'innovation, dont une grande partie sous forme de «fonds de fonds », comme les fonds de capital-risque sur les technologies de l'information, les biotechnologies, les écotechnologies... Vient s'ajouter une partie d'investissements en direct dans les PME par le fonds Ecotech (150 millions $€$ ), volume assez correct en matière de fonds de capital-risque.

\section{Les marchés, les concurrences, les perspectives}

Sylvie Alexandre, coordonnatrice du récent rapport sur les usages non alimentaires de la biomasse, a rappelé un constat établi : les grandes prospectives disponibles sur les scénarios alimentaires à 2050 sont toutes convergentes, à l'opposé des prospectives énergétiques qui, elles, divergent sur les trajectoires en termes de sobriété, et en parts d'énergies renouvelables, notamment hydro-carbonées. Le sujet est donc toujours mal cerné par les modélisations, et il y a encore de la place pour des travaux de prospective. Il ressort toutefois, quels que soient les scénarios, une double exigence majeure de sobriété pour les modes alimentaires (e.g. protéines animales) et pour les consommations énergétiques (directes et indirectes). Ceci suppose donc, en particulier en Europe, le besoin d'une capacité des pouvoirs publics à assurer des évaluations comparatives et évolutives de performances entre les différentes filières, et de rechercher une meilleure convergence intra-communautaire.

La sobriété et l'efficacité énergétiques constituent le principal métier de Dalkia France (filiale de EDF et de Veolia) : réseaux de chaleur, récupération de chaleur, biomasse, géothermie). Son directeur général, Francis Habègre, rappelle que l'énergie thermique est un "métier de pauvre», qui ne peut de ce fait, dans la biomasse-chaleur, concurrencer le bois d'œuvre ou le bois d'industrie, dont la valeur ajoutée est bien supérieure à celle de la «chaleur ». En revanche, la valorisation énergétique de la biomasse-sous produit apporte une valeur complémentaire et de la synergie aux forestiers et à la filière bois. Le pragmatisme amène à réfléchir «local» pour limiter les transports et les coûts, avec des plans d'approvisionnements validés par les préfets ou par des autorités compétentes. La plaquette de bois, marché en croissance, représente ainsi déjà plus de $50 \%$ de l'approvisionnement total de Dalkia, qui prévoit de tripler encore le volume de bois utilisé en chaufferies (à 3 millions de tonnes/an) en 2015.

Sylvie Alexandre a complété le propos en confirmant effectivement que la valeur ajoutée est d'abord le fait de la transformation du bois d'œuvre ou du bois-fibres en matériau, et qu'il faut développer une vision cohérente, synergique et systémique, et trouver une cohérence entre le stockage de carbone en forêt et la valorisation de la forêt. On pourrait certes valoriser toute la production de bois en énergie mais avec si peu de valeur ajoutée et d'emplois induits que cela ne permettrait pas d'assurer la pérennité des investissements en forêt, ni la protection de ses peuplements. Dès aujourd'hui, le problème est d'ailleurs posé et il faudrait déjà inventer de nouveaux mécanismes (dont une rémunération du carbone absorbé et stocké, peut être) pour inciter les propriétaires à couper et reboiser. . Le bois ne paye plus, seul, le renouvellement de la forêt, et un autre modèle économique reste à trouver.

Pour Stanislas Bouchard, directeur général de Cristal Union, et dans la perspective des biocarburants de seconde génération à base cellulosique, il ne faut pas séparer les produits agricoles de ceux de la forêt. Ces deux secteurs doivent impérativement communiquer. La France et l'Allemagne ont ainsi des manières très différentes de se chauffer, qui peuvent influer sur la façon dont la biomasse va se développer en Europe. Il y a des symptômes alarmants : dans certains pays (ex. Belgique), on importe des granulés de bois ou de sciure pour faire de l'électricité, parfois même sans valoriser la chaleur coproduite ! C'est peu efficace, c'est même un gaspillage de bioressources, et il n'est même pas certain que les ACV soient meilleures qu'avec du fioul.... Les allemands soutiennent par ailleurs fortement la méthanisation, avec 4 milliards $€$ d'aides par an (800 000 ha de maïs y sont consacrés!), ce qui équivaut de fait à une seconde PAC et ce qui n'est précisément pas neutre dans les négociations sur la PAC. La question pour la France devient alors : veut-on être le «réservoir à bois » de l'Europe ou veut-on le valoriser chez nous?

Caroline Rayol, ancienne conseillère du gouvernement brésilien en politiques publiques et en bio-énergies, a informé l'assemblée sur les dynamiques de développement de la bioéconomie au Brésil, où la priorité est de réduire les inégalités entre le pays «côtier», créateur apparent d'emplois et de richesses, et l'arrière-pays où prédomine encore la petite agriculture. La bio-économie apparaît sans conteste comme une opportunité pour développer le Brésil globalement et de façon équilibrée, en recherchant une synergie entre les différents marchés pour la biomasse : coopération entre filières et complémentarités non destructives. Les biocarburants sont ainsi, naturellement, au cœur de la stratégie de développement en filière, sans que la petite agriculture en soit écartée : le Brésil en est excédentaire et exporte du bio-éthanol $(50 \%$ vers les USA, $25 \%$ vers la Corée du Sud et $25 \%$ vers le Japon). La production, qui était de 23 milliards de litres en 2011, est prévue à 73 milliards de litres en 2020, avec l'ambition d'exporter 6,3 milliards de litres. Pour le gouvernement brésilien, il n'y a en outre aucune concurrence, et aucune solution de continuité, entre les différentes générations de biocarburants comme on semble le croire à tort en Europe ! La G1 et la G2 sont et resteront complémentaires et interdépendantes. Et pour le Brésil, si la G2 se développe un jour (ce qui n'est pas certain), cela ne veut pas dire pour autant que la G1 aurait fini d'exister...

Les perspectives en matière de chimie verte ont été dressées à son tour par Nicolas de Warren, directeur des relations institutionnelles d'Arkema, qui a commencé par illustrer le potentiel des produits bio-sourcés avec le cas du Rilsan. Le Rilsan est un polymère technique renouvelable (matières plastiques et fibres synthétiques) à très haute valeur technique 
produit dès 1950 à partir de graines de ricin importées d'Inde. Il y a 5 ans, la révision de la politique marketing d'Arkema, en mettant en avant l'aspect bio-sourcé des produits, a permis de passer d'une croissance du Rilsan de l'ordre de $10 \%$, à $20 \%$ par an! Arkema traite d'ailleurs globalement d'importants volumes de bio-ressources en Europe (amidons, huiles... ). Il y a 6 ans, Arkema prévoyait un objectif de croissance de $15 \%$ en 2020-2030, mais a dû revoir cet objectif à la baisse. En effet, la place de la Chine, qui réalisait $8 \%$ de la chimie mondiale en 1995, évolue rapidement : $34 \%$ de part de marché aujourd'hui et l'on prévoit $54 \%$ en 2025 ! D'autre part, le coût de l'énergie pèse lourd : le coût d'une tonne de polyéthylène produit sur du gaz américain à 4 \$/million de BTU est de $30 \%$ inférieur à celui du polyéthylène produit à partir d'éthylène européen. L'écart atteint 300 \$ par tonne. La perspective d'une croissance mondiale continue des prix du gaz est en train de s'inverser, peut être à cause du gaz de schiste! L'UE risque alors de ne pas pouvoir tenir la concurrence. Il s'agit d'un changement structurel pour toutes les filières d'aval de la chimie, y compris la chimie du végétal. Les filières bio-sourcées ont alors une place concurrentielle à jouer en Europe à condition de ne pas s'engager sur le marché des commodités, mais de se placer au contraire sur les filières à forte ou très forte valeur ajoutée, avec de la recherche, de l'innovation, de l'intégration...

À propos d'un autre exemple enfin, Arkema produit 300000 tonnes d'acide acrylique par an en France, d'origine pétrolière. Pour remplacer la matière première par du glycérol bio-sourcé, Arkema compte sur le biodiesel et sur son sous produit de process, la glycérine. La mise au point du procédé avance bien, mais Arkema se pose la question de passer ou non au stade de la démonstration industrielle, et quand ? Où trouver et se garantir notamment des approvisionnements pour des quantités annuelles à hauteur de 350000 tonnes de glycérol ? En Europe, et notamment en France ? Au Brésil ? Il faut arriver à montrer l'intérêt de la chimie bio-sourcée dans une logique industrielle globale, avec des indicateurs, et en précisant bien de quoi on parle aux actionnaires : approche globale, visibilité, fiabilité des politiques et des prix, traçabilité, concentration ou diversification des sites industriels?

\section{Les mises en perspectives}

Bernard Chevassus-au-Louis, ancien président du Museum d'Histoire naturelle, a posé la question de ce qui se joue dans les évolutions du vocabulaire. Croissance, développement, éco-développement, développement durable, économie verte, croissance verte, bio-économie : que devient le pilier social dans toute cette sémantique? Quelle est la place des aspects liés à l'emploi, mais aussi à l'appropriation des choix et des décisions par les sociétés? Est-ce que le carbone vert va être plus «vert» que la « révolution agricole verte d'après guerre », qui a eu des résultats remarquables, mais aussi des impacts induits moins désirables? Les bio-pesticides peuvent aussi poser des problèmes environnementaux, tout comme le bois énergie provoque des émissions de micro-particules... Les méthodes actuelles d'ACV peinent à prendre en compte tous ces impacts, comme elles ignorent souvent la spécificité du cycle naturel du carbone! On parle beaucoup de biomasse, mais peu de bio-information. Le vivant, c'est aussi de l'information qui circule : les êtres vivent d'échanges et de compréhensions mutuelles, et il y a peut-être là des idées pour réguler le système. Par exemple, sur la question des première et deuxième générations de biocarburants et de chimie du végétal... si la prudence s'impose, un peu d'audace et de bon sens ne nuit pas pour autant. Précaution ne veut pas dire immobilisme. Quand on présente une pièce brillante à la société, elle demande aussi à voir l'autre face, il faut prendre en compte cette demande.

Pascal Picq, paléoanthropologue, maître de conférences au Collège de France, a mis enfin en perspective les débats dans la dimension de l'évolution humaine, remarquant que le titre du colloque, «l'économie verte au service de la croissance... » a suscité des commentaires par rapport à la décroissance et au progrès. Les questions d'écologie suscitent parfois des réactions comme « on va retourner aux cavernes». Cela donne l'impression qu'il n'y a pas d'autre modèle que le nôtre, et qu'on extrapole, mais l'évolution ce n'est pas cela : à chaque époque, ses contraintes, ses expériences, ses échecs, ses succès, qui font une diversité, une évolution...

Les avancées en sciences ne reposent pas que sur les observations et les expérimentations, mais aussi par changements de paradigmes. Par exemple, l'invention de la systématique : observer, comparer, classer... démarche qui est celle de Linné. Puis, la question « est-ce que cette liste ne raconte pas une histoire? » mène aux théories de l'évolution.

Les débats de ce colloque relèvent de ce changement de paradigme en cours, mais il faut aussi écouter les signaux faibles : comment se fait-il que des personnalités scientifiques aient contesté aussi longtemps le changement climatique, et qu'une société d'auditeurs soit prête à écouter un discours ou non, aussi peu fondé ou séduisant soit-il ?

En définitive, tous les intervenants touchent à la biologie. Depuis deux siècles, les biologistes ont une assez bonne perception des enjeux. Comment concevoir les changements et l'évolution? Nous avons tous gardé l'idée que l'invention est fille de la nécessité. Mais il ne suffit pas d'un colloque pour susciter l'innovation. Rappelons-nous «l'invention» de l'agriculture : changements de société, de philosophie, de religion, de systèmes politiques. Cela a pris des siècles... alors que nous avons seulement en moyenne quarante ans dans cet amphithéâtre. La diversité, la perte de diversité... Un livre ${ }^{6}$ : «l'homme peut-il s'adapter à lui-même? » Le vrai terme est la co-évolution : une espèce n'évolue pas seule, mais avec d'autres espèces. La biodiversité nous apprend une chose : depuis 4 milliards d'années, il y a sur Terre la même quantité de matière vivante qui se renouvelle sans cesse, mais avec des écosystèmes différents. On a souvent été dans la journée, dans ce colloque, sur des termes de concurrence... mais il y a des écosystèmes qui sont plus riches que d'autres. On juge la rentabilité des molécules organiques à l'aune de celles du pétrole... il faut penser autrement.

Il y a des structures (Ademe, IFP Énergies nouvelles...), et des expériences fort intéressantes. En Basse-Normandie, on

\footnotetext{
6 Jean-François Toussaint, Bernard Swynghedauw, Gilles Bœuf. Editions Quae 2012.
} 
voit se développer par exemple une collectivité d'échanges et de service dans le monde agricole, avec des enjeux d'aujourd'hui.

«On a toujours fait comme ça ». Non, mais on est très conservateurs dans les domaines qu'on ne connaît pas. Comment faire ? C'est le problème de la culture entrepreneuriale. L'économie ignore les entreprises... Il n'y a pas de vraie culture entrepreneuriale en France : on forme nos étudiants pour faire carrière dans des grosses structures d'emploi. Mais les grosses structures ne font pas les mêmes inventions que les petites, et les vraies innovations de rupture ne viennent pas souvent des grandes entreprises. Il faut accepter que des gens entreprennent et échouent.

Il faut accepter que la bio-économie « tente le futur »!

Cite this article as: Étienne Pilorgé, Claude Roy. Colloque «l'économie verte au service de la croissance ». OCL 2013, $20(5)$ A601. 